\title{
A Randomized Controlled Trial of FNB versus FICB for Patients with Femoral Neck Fractures Before Spinal Anesthesia
}

This article was published in the following Dove Press journal:

Clinical Interventions in Aging

\author{
Yi Liang' \\ Lv Lv ${ }^{2}$ \\ Liang $\mathrm{He} \mathbb{D}^{\prime}$ \\ Wei Deng $\mathbb{B D}^{3}$ \\ Cai Chen' \\ Jingjuan $\mathrm{Li}^{1}$
}

'Department of Anesthesiology, The Affiliated Hospital of Guilin Medical University, Guilin, People's Republic of China; ${ }^{2}$ Emergency Department, The Affiliated Hospital of Guilin Medical University, Guilin, People's Republic of China; ${ }^{3}$ Department of Anesthesiology, The First Hospital of Jiaxing (Affiliated Hospital of Jiaxing University), Jiaxing, People's Republic of China
Correspondence: Liang $\mathrm{He}$ Department of Anesthesiology, The Affiliated Hospital of Guilin Medical University, Lequn Road I5\#, Guilin 54I00I, People's Republic of China Tel +8613659633168

Email jimyliang@hotmail.com
Background and Objectives: Patients with femoral neck fractures often suffer severe pain. This randomized controlled clinical study compared the effect of femoral nerve block (FNB) and fascia iliaca compartment block (FICB) in this population.

Patients and Methods: Forty-six patients were randomly assigned to one of the two groups: FNB group (femoral nerve block, $n=23$ ) or FICB group (fascia iliaca compartment block, $n=23$ ). Before positioning for spinal anesthesia, patients received FNB with $15 \mathrm{~mL}$ of $0.5 \%$ ropivacaine or FICB with $40 \mathrm{~mL}$ of $0.5 \%$ ropivacaine. Pain was evaluated using a visual analogue scale (VAS) at rest and during hip flexion after admission to the operation room; at 3, 5, 8, and 10 min after analgesia intervention; and during positioning for spinal anesthesia. Positioning was attempted after $10 \mathrm{~min}$ of analgesia intervention in each group. Time required to perform spinal anesthesia, quality of positioning, and patient satisfaction were documented.

Results: The VAS scores in the FNB group were significantly lower than those in FICB group at 3 and $5 \mathrm{~min}$ after analgesia intervention $(\mathrm{P}=0.000)$. However, there were no significant differences in VAS between groups at 8 or 10 min or during positioning.

Conclusion: FNB and FICB produce similar analgesic effects in patients with femoral neck fractures, but FNB has a more rapid onset of pain relief.

Keywords: femoral neck fracture, femoral nerve block, fascia iliaca compartment block, analgesia

\section{Introduction}

As the average life span of the Chinese population increases, the incidence of femoral neck fractures in this population has also grown, with these patients usually requiring surgical hip repair. Although spinal anesthesia is normally used during femoral neck fracture repair, patients may experience extreme pain due to the slight overriding of fracture ends during the positioning process for spinal anesthesia, ${ }^{1}$ which can lead to severe patient distress and sympathetic activation that manifests as tachycardia, hypertension, and increased cardiac work. To alleviate pain and improve positioning before anesthesia, non-steroidal anti-inflammatory drugs (NSAIDs), intravenous opioids, or peripheral nerve blockades (PNBs) are used. However, the analgesic effect of NSAIDs is limited and may cause gastrointestinal bleeding and platelet inhibition. In addition, opioids are effective in controlling static rather than dynamic pain, and opioids do not provide sufficient dynamic state pain relief. $^{2}$ Most importantly, these patients are often elderly with delicate physical 
conditions, rendering them more susceptible to the side effects of opioids such as nausea, vomiting, sedation, and respiratory depression. ${ }^{3}$ A systematic review revealed that regional nerve blocks in patients with hip and femoral neck fractures can reduce pain and decrease the need for intravenous opiates. ${ }^{4}$ Similarly, Sia and colleagues compared the analgesic effects of intravenous fentanyl with those of femoral nerve block (FNB) using lidocaine and showed that FNB provided better pain relief, ${ }^{5}$ while Kumar et al evaluated the sensory blockade in the lateral, anterior, and medial parts of the thigh and concluded that ultrasound-guided fascia iliaca compartment block (FICB) provided good pain management. ${ }^{6}$ Couto and colleagues reported that FICB significantly reduced the pain in the preoperative period, as indicated by visual analogue scale (VAS) scores. ${ }^{7}$

To date, the analgesic efficacy and onset time of ultrasound-guided FICB while positioning patients with femoral neck fracture have not been directly compared with those of ultrasound-guided FNB. This prospective randomized study compares pain management and onset time of FNB and FICB while positioning patients for spinal anesthesia before femoral neck fracture repair.

\section{Patients and Methods}

This trial was reviewed and approved by the Ethics Committee at the Affiliated Hospital of Guilin Medical University. Written informed consent was obtained from all participants before their recruitment in the study. This trial was conducted in accordance with the Declaration of Helsinki and registered at the Chinese clinical trial registry website (registration number ChiCTR1800017164).

Between August 2018 and June 2019, we recruited patients aged 59-86 years with American Society of Anesthesiologists (ASA) physical status I-III and Garden's III or IV femoral neck fractures who were scheduled to undergo partial or full hip displacement surgery. Exclusion criteria included dementia, impaired cognitive function, central nervous system disorder, peripheral neuropathy, allergy to amide local anesthetics, puncture site infection, multiple fractures, or any pain management in $12 \mathrm{~h}$ before surgery.

The use of a 10-cm VAS ( 0 indicating no pain and 10 indicating maximal pain) was explained to patients after they entered the operating room to ensure that they completely understood it. In the operating room, intravenous access was established, and Ringer's lactate injection was infused at $5 \mathrm{~mL} \cdot \mathrm{kg}^{-1} / \mathrm{h}$. A face mask was used to provide supplemental oxygen $\left(6 \mathrm{~L} \cdot \mathrm{min}^{-1}\right)$. Pulse oximetry data, electrocardiography findings, blood pressure, and urinary output were monitored. No sedative or analgesic medications were given to patients.

FNB was performed under the guidance of high-frequency linear ultrasonography (Sonosite Edge II, Sonosite, Bothell, WA, USA). The ultrasound probe was placed in transverse orientation on the upper thigh just inferior to the inguinal ligament. The femoral nerve is lateral to the femoral artery and deep to the fascia iliaca and appears hyperechoic. After disinfection of the skin in the inguinal region and upper thigh, a 22-G, 50-mm, short-bevel Stimuplex D needle (B. Braun, Melsungen, Germany) was inserted at the lateral side of the thigh and $1 \mathrm{~cm}$ outside the edge of the probe. From the outside to the inside, toward the femoral nerve, the needle was inserted using the in-plane technique. Once the tip of the needle was close to the nerve, negative aspiration was performed, and a multiple-injection technique was used with the tip of the needle in several positions around the femoral nerve until $15 \mathrm{~mL}$ of $0.5 \%$ ropivacaine had been administered.

Although we performed FICB in the inguinal region and used the same ultrasonography probe and puncture needles as described for FNB, there were some differences. In FICB, the ultrasound probe was placed in transverse orientation on the thigh just inferior to the inguinal ligament at lateral to the femoral artery. The two fascial planes - the fascia lata and fascia iliaca-were visualized as two hyperechoic lines. The needle was inserted in-plane, and the needle tip was visualized penetrating the fascia lata followed by the fascia iliaca. After puncturing the fascia iliaca and negative aspiration, 40 $\mathrm{mL}$ of $0.5 \%$ ropivacaine was injected. An expanding anechoic collection between the fascia iliaca and iliopsoas muscle served as visual confirmation of correct local anesthetic placement.

Both FNB and FICB were performed by a very experienced anesthesiologist, and local anesthetic injection was immediately stopped when the patient had any discomfort. Ten minutes later, they were placed laterally with the fractured leg on the top for spinal anesthesia. VAS scores of patients on rest and movement were evaluated before FNB or FICB; 3, 5, 8, and 10 min after analgesic intervention; and during positioning. When a patient reported a VAS $>4$ during positioning, procedure was stopped, and $1.5 \mu \mathrm{g} \cdot \mathrm{kg}^{-1}$ intravenous fentanyl was given. Positioning was reattempted after reducing VAS to $\leq 4$.

Spinal anesthesia was administered by another anesthesiologist in the L2-L3 or L3-L4 intervertebral space using 
a 25-G Quincke spinal needle with a midline approach. Appropriate intrathecal injection was administered after confirming free flow and positive aspiration for cerebrospinal fluid, 2.5 to $3 \mathrm{~mL}$ of $0.5 \%$ levobupivacaine was administered intrathecally. The sensory block level was controlled to no more than the T8-T10 level. Hypotension (systolic blood pressure $<90 \mathrm{mmHg}$ or a $>30 \%$ drop from baseline) was treated with ephedrine (10-15 mg). Bradycardia (heart rate $<50$ beats per min) was treated with atropine (0.3-0.5 mg). Respiratory depression (pulse oxygen saturation $<90 \%$ or a $>10 \%$ drop from baseline) was treated with face mask oxygen inhalation.

The primary outcome was patient VAS at rest and on movement (before analgesic intervention; 3, 5, 8, and 10 min after analgesic intervention; and during positioning). The secondary outcomes were the quality of patient positioning for spinal anesthesia (recorded on a scale of 0 to 3 : 0 , unsatisfactory; 1 , satisfactory; 2 , good; 3 , optimal), time to perform spinal anesthesia (from the start of positioning to levobupivacaine injection completion), and patient satisfaction (on the second postoperative day, patients were asked whether they would choose the same anesthetic procedure: "yes" or "no"). The study was blinded from the aspect of the patients; the anesthesiologist who administered spinal anesthesia; and the observer who recorded VAS scores, quality of patient positioning for spinal anesthesia, time to perform spinal anesthesia, and patient satisfaction.

Preliminary experiments revealed that FNB achieved a lower VAS score than FICB at $5 \mathrm{~min}$ after analgesic intervention. The test level $\alpha$ was set as 0.05 , and the power level 1- $\beta$ was set as 0.9 . Considering a significant difference at a mean difference of 2.1 in the VAS score, with a standard deviation (SD) of 2.1, the sample size in each group was estimated to be 23 . Thus, a total of 50 patients were enrolled.

Statistical analysis was performed using Statistical Product for Social Sciences (SPSS) software Version 18.0 (SPSS Inc., Chicago, IL, USA). Continuous variables are presented as mean $\pm \mathrm{SD}$, and in case of normal distribution, analyzed using independent $t$-tests, whereas MannWhitney $U$-tests were performed for nonnormally distributed variables. Repeated measure analysis of variance was used to compare VAS scores within groups. The $\chi^{2}$ test was used to compare the quality of positioning and patient acceptance. $\mathrm{P}<0.05$ was considered statistically significant.

\section{Results}

Over an 11-month period, 50 patients were assessed for eligibility, and 46 were enrolled in and completed the study (Figure 1). Demographic characteristics and surgical and anesthesia data are shown in Table 1.

There were no significant differences between the FNB and FICB groups in static $(\mathrm{P}=0.897)$ or dynamic pain score $(\mathrm{P}=0.657)$ before analgesic intervention. Both static and dynamic VAS scores significantly decreased in both groups following either FNB or FICB (Figures 2 and 3).

At 3 and 5 min after analgesic intervention, the FNB group reported significantly lower VAS scores than the FICB group $(\mathrm{P}=0.000)$. There were no significant VAS score differences between the groups at 8 and $10 \mathrm{~min}$ after analgesic intervention or during positioning (all $\mathrm{P}>0.05)$.

Intragroup comparison showed no significant differences in VAS scores in the FNB group at 5, 8, and 10 min after analgesia intervention $(\mathrm{P}>0.05)$. But at $8 \mathrm{~min}$ after analgesic intervention, VAS scores in the FICB group continued to decline compared with those at $5 \mathrm{~min}$. There were no significant differences in VAS scores at 8 and 10 min after analgesic intervention in the FICB group $(\mathrm{P}>0.05)$.

The time to perform spinal anesthesia was very short in both groups, the quality of positioning for spinal anesthesia performance was relatively high, and patient satisfaction was also relatively high. There were no significant differences between groups for any of these parameters (all $\mathrm{P}>0.05$, Table 2).

There were no statistically significant differences between groups in blood pressure, heart rate, urinary output, and fluid and vasoconstrictor consumption. No adverse effects of ropivacaine were noted. No cases of vascular puncture or paresthesia were observed in either group.

\section{Discussion}

FNB and FICB are two commonly used PNB methods in patients with femoral neck fractures. In this study, we compared the use of FNB and FICB under ultrasound guidance and that both approaches provide good pain management. More importantly, FICB and FNB had similar effects in alleviating pain caused by preoperative postural changes in patients with femoral neck fractures, but those who underwent FNB had faster analgesic onset and required less local anesthetics. 


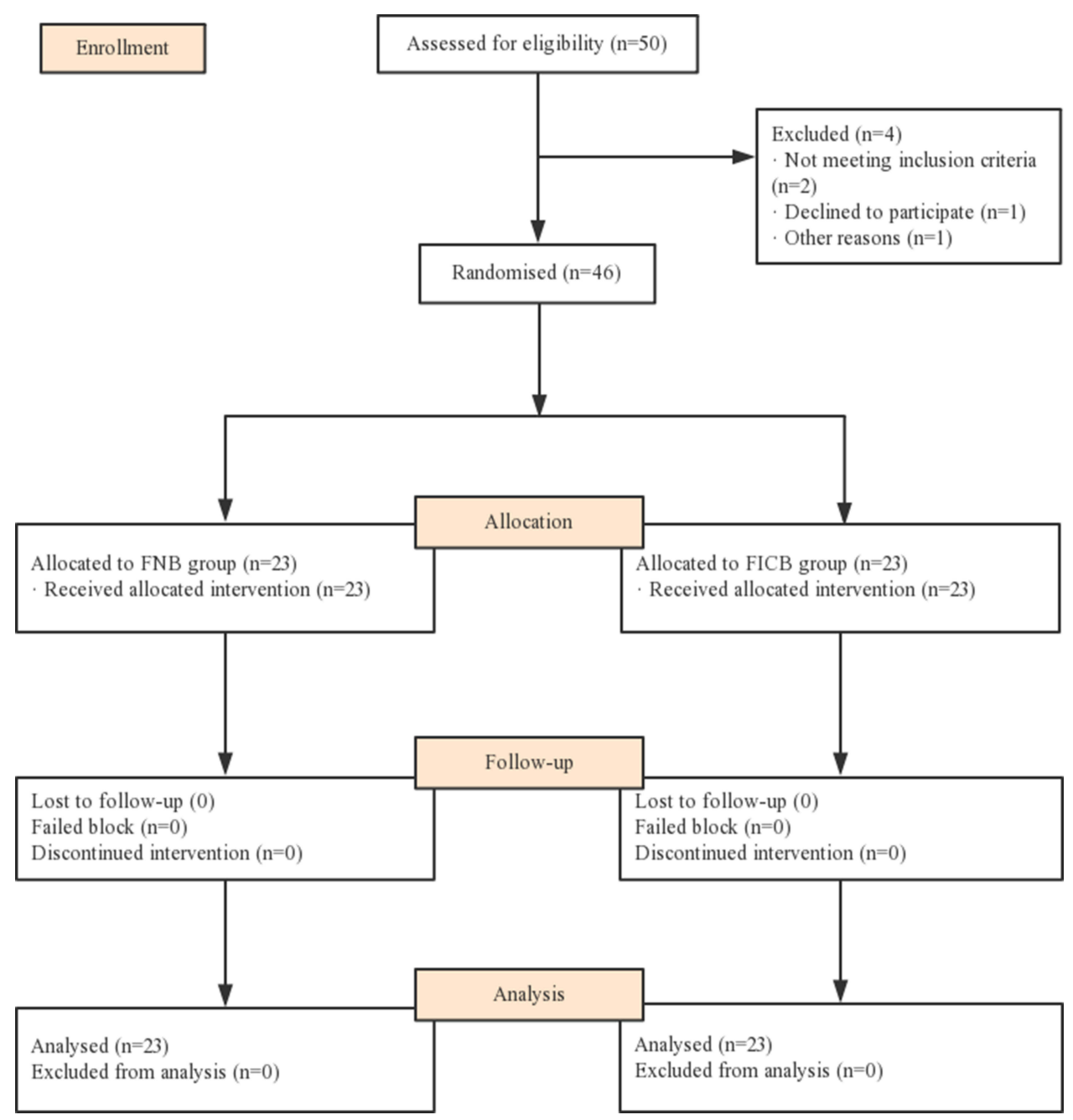

Figure I Consort flowchart of the study.

The hip joint is innervated by the femoral nerve, sciatic nerves, obturator nerve, and superior gluteal nerve; the main innervation includes the obturator nerve and femoral and

Table I Demographic Characteristics and Surgical and Anesthesia Data

\begin{tabular}{|l|l|l|}
\hline & $\begin{array}{l}\text { FNB } \\
\text { Group }\end{array}$ & $\begin{array}{l}\text { FICB } \\
\text { Group }\end{array}$ \\
\hline ASA (II/III), $\mathrm{n}$ & $17 / 6$ & $17 / 6$ \\
Sex (M/F), $\mathrm{n}$ & $6 / 17$ & $7 / 16$ \\
Age (year) & $74.3 \pm 8.2$ & $73.9 \pm 7.8$ \\
Height (cm) & $158.4 \pm 7.9$ & $157.4 \pm 6.8$ \\
Weight (kg) & $55.6 \pm 9.5$ & $55.0 \pm 11.8$ \\
Time from fracture to surgery & $2.2 \pm 1.0$ & $2.1 \pm 0.9$ \\
(days) & & \\
\hline
\end{tabular}

Abbreviations: ASA, American Society of Anesthesiologists grade; FICB, fascia iliaca compartment block; FNB, femoral nerve block.

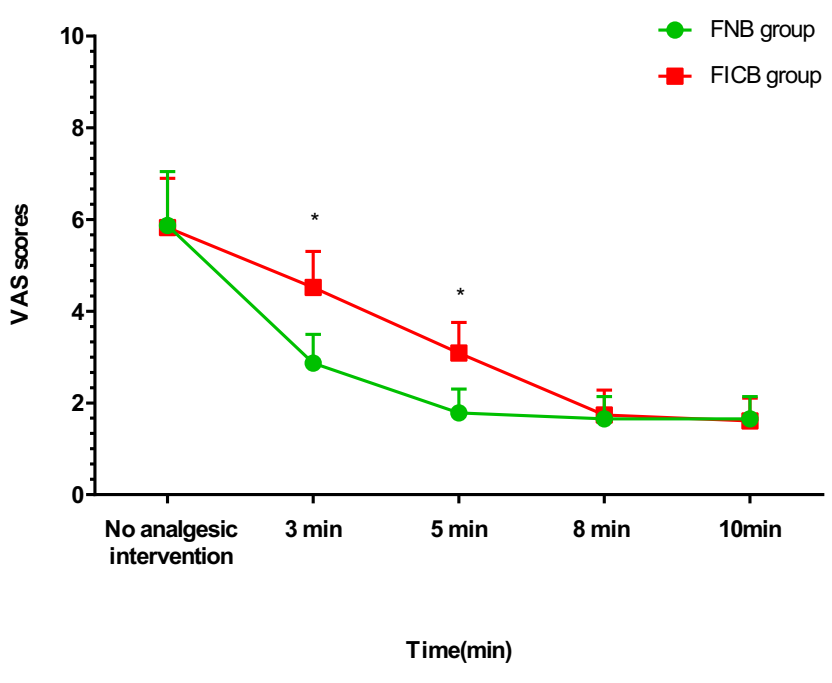

Figure 2 Static VAS scores in each group. ${ }^{*} \mathrm{P}<0.05$, FNB group vs $\mathrm{FICB}$ group. 


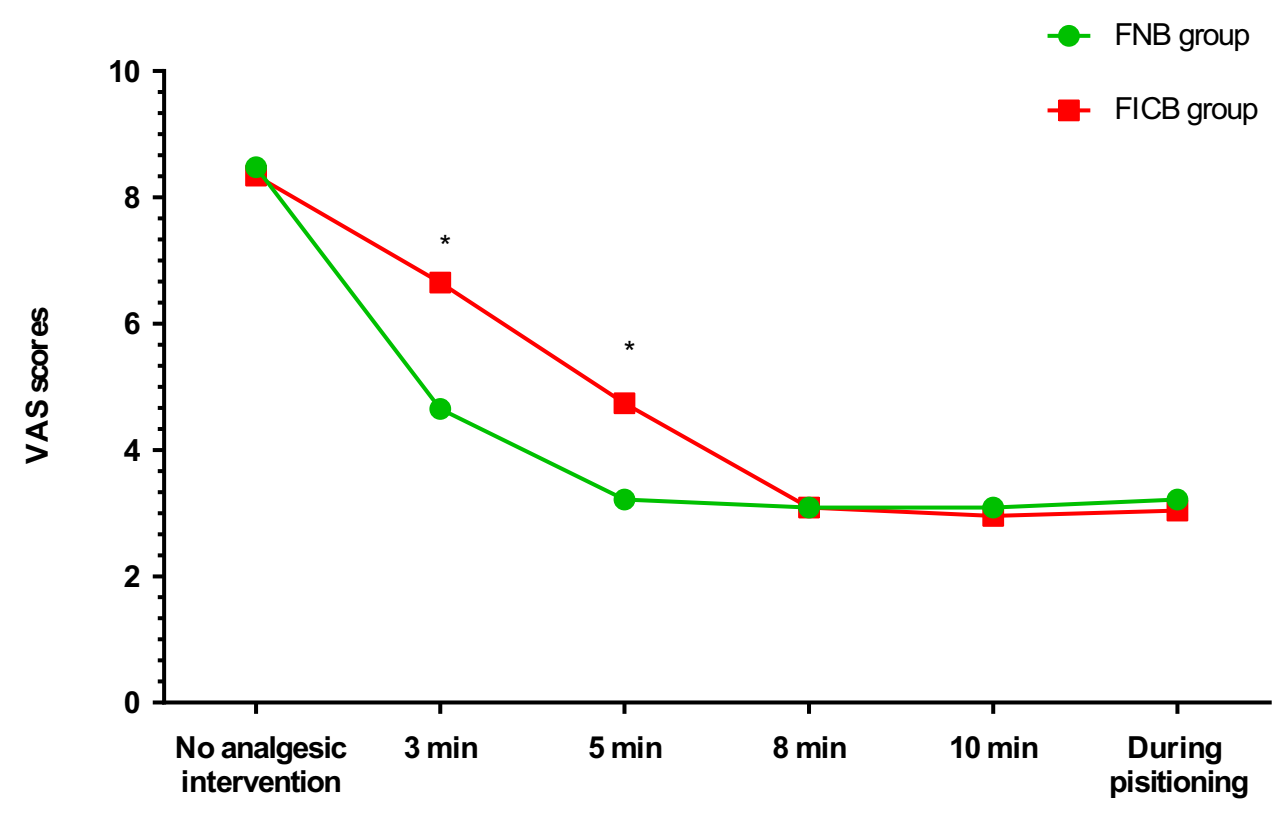

Time(min)

Figure 3 Dynamic VAS scores and VAS scores during positioning. ${ }^{*} \mathrm{P}<0.05$, FNB group vs FICB group.

sciatic nerves. ${ }^{8}$ An early report from 2000 stated that FICB could simultaneously block the femoral nerve, lateral femoral cutaneous nerve (LFCN), and anterior branch of the obturator nerve. ${ }^{9}$ Bouaziz and colleagues thought that these studies may have mistaken an FNB for an obturator nerve block when the cutaneous distribution of the obturator nerve was assessed on the medial aspect of the thigh, ${ }^{10}$ and they pointed out that the only way to effectively evaluate obturator nerve function is to assess adductor strength. A recent study by Swenson et al confirmed that FICB produced a reliable clinical effect on the femoral nerve and LFCN. However, injections did not produce obturator nerve block either at the level of the retroperitoneum or the inguinal ligament. ${ }^{11}$ Since the $\mathrm{LFCN}$ is the only nerve other than the femoral nerve to be blocked by FICB and the LFCN provides no innervation to the hip, there is no theoretical benefit to FICB over FNB.

Table 2 Performance Times, Quality of Positioning, and Patient Satisfaction

\begin{tabular}{|l|l|l|}
\hline & FNB Group & FICB Group \\
\hline Time to perform SA (min) & $2.83 \pm 0.79$ & $2.78 \pm 0.67$ \\
Quality of positioning (0/I/2/3), n & $0 / 6 / 13 / 4$ & $0 / 5 / 14 / 4$ \\
Patient satisfaction (yes/no), n & $21 / 2$ & $20 / 3$ \\
\hline
\end{tabular}

Abbreviations: FICB, fascia iliaca compartment block; FNB, femoral nerve block; SA, spinal anesthesia.
FICB and FNB have similar analgesic effects in relieving pain caused by changes in preoperative postural position in patients with femoral neck fractures. Based on the above viewpoints, the purpose of the present study was to compare the analgesic effect and onset time of FNB and FICB in patients with femoral neck fractures during positioning.

In the past, without the guidance of ultrasound, FICB was preferred to alleviate pain because the traditional FICB approach uses a puncture point distant from the neurovascular sheath and is simple and easy to perform. ${ }^{12}$ In addition, a series of risks such as vascular puncture, hematoma, nerve damage, infection, and intravascular injection may occur during the FNB. ${ }^{13}$ However, the increased use of ultrasound guidance has greatly reduced the risks associated with FNB. The literature suggests that ultrasound-guided FNB has a shorter onset time and is safer and more effective than FNB administered with a nerve stimulator or blindly, and the need for a local anesthetic was lower. ${ }^{14}$ We did not find any difference between the two methods in terms of vascular puncture and nerve damage.

The femoral nerve is derived from the lumbar plexus. Sensory innervation of the proximal femur and a varied portion of the intracapsular neck of the femur arise from the femoral nerve. ${ }^{15}$ In the inguinal ligament area, the femoral nerve is located $1-2 \mathrm{~cm}$ lateral to the femoral artery and vein and lies deeply under the fascia lata and fascia iliaca, between the fascia iliaca and iliopsoas muscle. ${ }^{13}$ 
When performing an FNB, we directly injected local anesthetic close to the nerve. However, when we performed an FICB, we injected local anesthetic between the fascia iliaca and iliopsoas muscle, and the femoral nerve was not blocked until the local anesthetic had spread through the connective tissue space to the femoral nerve. Obviously, FNB blocked the femoral nerve faster than FICB, which was confirmed by our results. FNB can reduce patients' waiting time in the operating room before spinal anesthesia, speed up operation room turnover, and reduce surgery time and cost.

An FICB is a volume-dependent block requiring 30-40 $\mathrm{mL}$ of local anesthetic in order to spread sufficiently through the sub-fascial compartment. ${ }^{16}$ However, 10 to $20 \mathrm{~mL}$ of local anesthetic is usually sufficient to block the femoral nerve during FNB. Taha and colleagues concluded that perineural injection of $15 \mathrm{~mL}$ of ropivacaine $0.167 \% \mathrm{w} / \mathrm{v}$ under ultrasound guidance can achieve successful FNB in $90 \%$ of patients. ${ }^{17}$

A study comparing FNB with FICB in patients with femoral neck fractures concluded that FNB provided better preoperative analgesia, ${ }^{18}$ possibly because the anesthetic volume in FICB (20 to $30 \mathrm{~mL}$ ) was inadequate to spread into the fascia iliaca compartment. However, Cooper and colleagues recently concluded that ultrasound-guided FNB is not superior to FICB, and both techniques achieved similar analgesic effects in patients with femoral neck or proximal femur fracture. ${ }^{19}$

Obviously, if patients with femoral neck fractures can receive PNBs in the emergency department or prior to transfer to the operation room, it will greatly alleviate pain, reduce opioid consumption and facilitate positioning during spinal anesthesia. PNBs for hip fractures are not widely used in China, which may be related to the lack of equipment and well-trained doctors, as well as the lack of public awareness of PNB safety and efficacy. All of these observations demonstrate that there is room for improvement.

Before positioning for spinal anesthesia, we assessed VAS scores only $10 \mathrm{~min}$ after analgesic intervention, whereas other studies employed longer waiting times in similar experiments to ensure that the local anesthetic reached its peak analgesic effect. ${ }^{1,20}$ Kumar and colleagues conducted a study with $30 \mathrm{~mL}$ of $0.5 \%$ ropivacaine and evaluated sensory blockade at 5, 10, and 20 min after performing FICB, and they concluded that sensory blockade at $20 \mathrm{~min}$ was the same as that at $10 \mathrm{~min}$ in all parts of the thigh. ${ }^{6}$ Therefore, we chose $10 \mathrm{~min}$ for of final time point. Nevertheless, this is a limitation of our study.
Another disadvantage of this study is that we chose a long-acting local anesthetic, ropivacaine, rather than a much faster onset local anesthetic such as lidocaine for a study evaluating the onset of analgesia. A study by Diakomi and colleagues showed that patients who received FICB with ropivacaine before spinal anesthesia experienced effective analgesia with no need for additional drugs on the first postoperative day. ${ }^{20}$ We chose ropivacaine for the purpose of alleviating postoperative pain.

When performing an FNB or FICB, different conclusions may be drawn depending on the local anesthetic used, concentrations and volumes, and different levels of ultrasound-guided nerve blockade. In general, FNB has the advantages of using less local anesthetics and a more rapid onset, which may be more desirable than FICB.

\section{Conclusions}

Both FNB and FICB provide good pain management in patients with femoral neck fractures. While both approaches produce similar analgesic effects, FNB has a faster onset with lower local anesthetic requirement.

\section{Abbreviations}

FICB, fascia iliaca compartment block; FNB, femoral nerve block; PNB, peripheral nerve blockade; VAS, visual analogue scale; LFCN, lateral femoral cutaneous nerve; NSAIDs, non-steroidal anti-inflammatory drugs; ASA, American Society of Anesthesiologists; SD, standard deviation; SPSS, Statistical Product for Social Sciences.

\section{Data Sharing Statement}

The individual de-identified participant data will not be shared by the authors following the publication.

\section{Ethics Approval and Informed Consent}

This trial was reviewed and approved by the Ethics Committee at the Affiliated Hospital of Guilin Medical University. Written informed consent was obtained from all participants before study recruitment. This trial was conducted in accordance with the Declaration of Helsinki and registered at the website of the Chinese clinical trial registry (registration number ChiCTR1800017164).

\section{Author Contributions}

All authors contributed to data analysis, drafting or revising the article, agree to the journal in which the paper was 
submitted, gave final approval of the version to be published, and agree to be accountable for all aspects of the work.

\section{Funding}

This study was supported by the National Natural Science Foundation of China (No. 81860239), the Guangxi Natural Science Foundation (Grant No. 2018GXNSFBA050062 and AD19110125), and the Guangxi Medical and Health Key Cultivation Discipline Construction Project.

\section{Disclosure}

The authors report no conflicts of interest in this work.

\section{References}

1. Yun MJ, Kim YH, Han MK, Kim JH, Hwang JW, Do SH. Analgesia before a spinal block for femoral neck fracture: fascia iliaca compartment block. Acta Anaesthesiol Scand. 2009;53:1282-1287. doi:10.1111/j.1399-6576.2009.02052.x

2. Williams H, Paringe V, Shenoy S, Michaels P, Ramesh B. Standard preoperative analgesia with or without fascia iliaca compartment block for femoral neck fractures. J Orthop Surg (Hong Kong). 2016;24:3135. doi:10.1177/230949901602400109

3. Rashid A, Beswick E, Galitzine S, Fitton L. Regional analgesia in the emergency department for hip fractures: survey of current UK practice and its impact on services in a teaching hospital. Emerg Med J. 2014;31:909-913. doi:10.1136/emermed-2013-202794

4. Ritcey B, Pageau P, Woo MY, Perry JJ. Regional nerve blocks for hip and femoral neck fractures in the emergency department: a systematic review. CJEM. 2016;18:37-47. doi:10.1017/cem.2015.75

5. Sia S, Pelusio F, Barbagli R, Rivituso C. Analgesia before performing a spinal block in the sitting position in patients with femoral shaft fracture: a comparison between femoral nerve block and intravenous fentanyl. Anesth Analg. 2004;99:1221-1224. doi:10.1213/01.ANE.00 00134812.00471 .44

6. Kumar D, Hooda S, Kiran S, Devi J. Analgesic efficacy of ultrasound guided FICB in patients with hip fracture. J Clin Diagn Res. 2016;10: UC13-UC16. doi:10.7860/JCDR/2016/17802.8123

7. Candal-Couto JJ, McVie JL, Haslam N, Innes AR, Rushmer J. Preoperative analgesia for patients with femoral neck fractures using a modified fascia iliaca block technique. Injury. 2005;36:505-510. doi:10.1016/j.injury.2004.10.015

8. Yu B, He M, Cai GY, Zou TX, Zhang N. Ultrasound-guided continuous femoral nerve block vs continuous fascia iliaca compartment block for hip replacement in the elderly: a randomized controlled clinical trial (CONSORT). Medicine (Baltimore). 2016;95:e5056. doi:10.1097/MD.0000000000005056

Clinical Interventions in Aging

\section{Publish your work in this journal}

Clinical Interventions in Aging is an international, peer-reviewed journal focusing on evidence-based reports on the value or lack thereof of treatments intended to prevent or delay the onset of maladaptive correlates of aging in human beings. This journal is indexed on PubMed Central, MedLine, CAS, Scopus and the Elsevie
9. Marhofer P, Nasel C, Sitzwohl C, Kapral S. Magnetic resonance imaging of the distribution of local anesthetic during the three-inone block. Anesth Analg. 2000;90:119-124. doi:10.1097/00000539200001000-00027

10. Bouaziz H, Vial F, Jochum D, et al. An evaluation of the cutaneous distribution after obturator nerve block. Anesth Analg. 2002;94:445449. doi:10.1097/00000539-200202000-00041

11. Swenson JD, Davis JJ, Stream JO, Crim JR, Burks RT, Greis PE. Local anesthetic injection deep to the fascia iliaca at the level of the inguinal ligament: the pattern of distribution and effects on the obturator nerve. J Clin Anesth. 2015;27:652-657. doi:10.1016/j. jclinane.2015.07.001

12. Dolan J, Williams A, Murney E, Smith M, Kenny GN. Ultrasound guided fascia iliaca block: a comparison with the loss of resistance technique. Reg Anesth Pain Med. 2008;33:526-531. doi:10.1016/j. rapm.2008.03.008

13. Layzell MJ. Use of femoral nerve blocks in adults with hip fractures Nurs Stand. 2013;27:49-58. doi:10.7748/ns2013.08.27.52.49.e7390

14. Mittal R, Vermani E. Femoral nerve blocks in fractures of femur: variation in the current UK practice and a review of the literature. Emerg Med J. 2014;31:143-147. doi:10.1136/emermed-2012-201546

15. Szucs S, Iohom G, O’Donnell B, et al. Analgesic efficacy of continuous femoral nerve block commenced prior to operative fixation of fractured neck of femur. Perioper Med (Lond). 2012;1(1):4. doi:10.1186/2047-0525-1-4

16. Hewson DW, Reddy R. Pre-operative femoral nerve block vs fascia iliaca block for femoral neck fracture - 6. Anaesthesia. 2013;68:1277-1278. doi:10.1111/anae.12498

17. Taha AM, Abd-Elmaksoud AM. Ropivacaine in ultrasound-guided femoral nerve block: what is the minimal effective anaesthetic concentration (EC90)? Anaesthesia. 2014;69:678-682. doi:10.1111/ anae. 12607

18. Newman B, McCarthy L, Thomas PW, May P, Layzell M, Horn K. A comparison of pre-operative nerve stimulator-guided femoral nerve block and fascia iliaca compartment block in patients with a femoral neck fracture. Anaesthesia. 2013;68:899-903. doi:10.1111/anae. 12321

19. Cooper AL, Nagree Y, Goudie A, Watson PR, Arendts G. Ultrasound-guided femoral nerve blocks are not superior to ultrasound-guided fascia iliaca blocks for fractured neck of femur. Emerg Med Australas. 2019;31:393-398. doi:10.1111/1742-6723. 13172

20. Diakomi M, Papaioannou M, Mela A, Kouskouni E, Makris A. Preoperative fascia iliaca compartment block for positioning patients with hip fractures for central nervous blockade: a randomized trial. Reg Anesth Pain Med. 2014;39:394-398. doi:10.1097/AAP.000 0000000000133
Bibliographic databases. The manuscript management system is completely online and includes a very quick and fair peer-review system, which is all easy to use. Visit http://www.dovepress.com/ testimonials.php to read real quotes from published authors. 